\begin{tabular}{c|c|c|} 
SELECCIONES MATEMÁTICAS \\
Universidad Nacional de Trujillo \\
ISSN: $2411-1783$ (Online) \\
2021; Vol. 8(2):316-325.
\end{tabular}

\title{
A class of surfaces with planar lines of curvature
}

\section{Una clase de superficies con líneas de curvatura planas}

\author{
Carlos M. C. Riveros (i) and Cid D. F. Machado
}

Received, Nov. 22, 2021

Accepted, Dec. 12, 2021

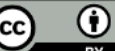

How to cite this article:

Riveros CMC, Machado CDF. A class of surfaces with planar lines of curvature. Selecciones Matemáticas. 2021;8(2):316325. http://dx.doi.org/10.17268/sel.mat.2021.02.08

\begin{abstract}
In this paper we study surfaces parameterized by lines of curvature. We obtain a characterization of a class of surfaces with planar lines of curvature, this characterization depend on certain holomorphic function. We present a class of surfaces with two family of planar lines of curvature, this class of surfaces includes the Dupin surfaces. Also, we give explicit examples.
\end{abstract}

Keywords . Planar lines of curvature, Dupin surfaces, holomorphic functions.

\section{Resumen}

En este articulo estudiamos superficies parametrizadas por líneas de curvatura. Obtenemos una caracterización de una clase de superficies con líneas de curvatura planas, esta caracterización depende de ciertas funciones holomorfas. Presentamos una clase de superficies con dos familias de líneas de curvatura planas, esta clase incluye las superficies de Dupin. También damos algunos ejemplos explícitos.

Palabras clave. Líneas de curvatura planas, superficies de Dupin, funciones holomorfas.

1. Introduction. A hypersurface $M^{n}$ is said to be Dupin if each principal curvature is constant along its corresponding surface of curvature. The Dupin hypersurface $M^{n}$ is said to be proper if the number $g$ of distinct principal curvatures is constant on $M^{n}$. Dupin hypersurfaces is a current research topic, we mention for example [6], [8], [10], [11], [15], [16] when the principal curvatures are all distinct, check that the curvature lines of a Dupin hypersurface are circles or straight lines, so the lines of curvature are planar curves.

Classify surfaces with planar curvature lines with a property additional geometry is a traditional and current research topic, for example according to Nitsche [9], minimal surfaces with planar lines of curvature in the Euclidian space was discovered by Bonnet and Enneper. In [2], the authors study surfaces with planar curvature lines and as a result, they stablish some characterization theorems for such surfaces. Moreover, they give a condition for a surface to be a surface of revolution.

In [7], the authors study surfaces with planar lines of curvature in the framework of Laguerre geometry and provide explicit representation formulae for these surfaces in terms of a potencial function. As an application, explicitly integrate all L-minimal surfaces with planar curvature lines. In [3] Leite, determine the orthogonal systems of cycles (curves of constant geodesic curvature) on the hyperbolic plane, aiming at the classification of maximal surfaces with planar lines of curvature in the Minkowski space.

Masal'tsev in [5] given a construction of surfaces in a three-dimensional Lobachevskii space $\mathbb{H}^{3}$ whose properties are similar to those of Joachimsthal surfaces in a three-dimensional Euclidean space; i.e., he gives a description of surfaces in $\mathbb{H}^{3}$ having one family of lines of curvature located in totally geodesic planes containing a common geodesic of $\mathbb{H}^{3}$. The author also proves that in $\mathbb{H}^{3}$, surfaces of constant mean

\footnotetext{
*Departamento de Matemática, Universidade de Brasília, 70910-900, Brasília-DF, Brazil. (carlos @mat . unb.br).

${ }^{\dagger}$ FacUnicamps, CEP 74535-280, Goiânia-GO, Brazil. (cidgeomat @ gmai l . com).
} 
curvature without umbilical points having one family of lines of curvature located in totally geodesic planes are surfaces of revolution (hyperbolic Delanoë surfaces). In [14], the authors given a characterization of hypersurfaces whose lines of curvature are planar. In [12], the authors study special classes of hypersurfaces parameterized by lines of curvature with some conditions on the Laplace invariants.

In this paper, using [4] we present surfaces parameterized by lines of curvature. We obtain a characterization of a class of surfaces with planar lines of curvature, this characterization depend on certain holomorphic function. We present a class of surfaces with two family of planar lines of curvature, this class of surfaces includes the Dupin surfaces. Also, we give explicit examples.

2. Preliminaries. Let $\Omega$ be an open subset of $\mathbb{R}^{n}$ and $u=\left(u_{1}, u_{2}, \cdots, u_{n}\right) \in \Omega$. Let $X: \Omega \subset \mathbb{R}^{n} \rightarrow$ $\mathbb{R}^{n+1}, n \geq 2$, be a hypersurface parametrized by lines of curvature, with distinct principal curvatures $k_{i}, 1 \leq i \leq n$ and $N: \Omega \subset \mathbb{R}^{n} \rightarrow \mathbb{R}^{n+1}$ be a unit normal vector field of $X$. Then

$$
\begin{aligned}
\left\langle X_{, i}, X_{, j}\right\rangle & =\delta_{i j} g_{i i}, 1 \leq i, j \leq n, \\
N_{, i} & =-k_{i} X_{, i},
\end{aligned}
$$

where the subscript ${ }_{i}$ denotes the derivative with respect to $u_{i}$. Moreover,

$$
\begin{aligned}
& X_{, i j}-\Gamma_{i j}^{i} X_{, i}-\Gamma_{i j}^{j} X_{, j}=0, \quad 1 \leq i \neq j \leq n, \\
& \Gamma_{i j}^{i}=\frac{k_{i, j}}{k_{j}-k_{i}}, \quad 1 \leq i \neq j \leq n,
\end{aligned}
$$

where $\Gamma_{i j}^{k}$ are the Christoffel symbols.

We now consider the higher-dimensional Laplace invariants of the system of equations (2.2) (see [1])

$$
\begin{aligned}
m_{i j} & =-\Gamma_{i j, i}^{i}+\Gamma_{i j}^{i} \Gamma_{i j}^{j}, \\
m_{i j k} & =\Gamma_{i j}^{i}-\Gamma_{k j}^{k}, \quad k \neq i, j, \quad 1 \leq k \leq n .
\end{aligned}
$$

Definition 2.1. A hypersurface $M^{n}$ is said to be Dupin if each principal curvature is constant along its corresponding surface of curvature.

The following Proposition obtained in [13] characterizes the hypersurfaces parametrized by planar lines of curvature.

Proposition 2.1. For $n \geq 2$, let $X: \Omega \subset \mathbb{R}^{n} \rightarrow \mathbb{R}^{n+1}$, be a hypersurface parametrized by lines of curvature, with distinct principal curvatures $k_{i}, 1 \leq i \leq n$.

The lines of curvature $\alpha_{i}\left(u_{i}\right)=X\left(u_{1}^{0}, u_{2}^{0}, \cdots, u_{i-1}^{0}, u_{i}, u_{i+1}^{0}, \cdots, u_{n}^{0}\right), 1 \leq i \leq n$ are planar if and only if

$$
k_{i, i} \Gamma_{i j}^{i}+k_{i} m_{i j}=0,1 \leq i \neq j \leq n .
$$

In this paper the inner product $\langle\rangle:, \mathbb{C} \times \mathbb{C} \rightarrow \mathbb{R}$ is defined by

$$
\langle f, g\rangle=f_{1} g_{1}+f_{2} g_{2}, \text { where } f=f_{1}+i f_{2}, g=g_{1}+i g_{2},
$$

are holomorphic functions.

In the computation we use the following properties: If $f, g: \mathbb{C} \rightarrow \mathbb{C}$ are holomorphic functions of $u=$ $u_{1}+i u_{2}$. Then

$$
\langle f, g\rangle_{, 1}=\left\langle f^{\prime}, g\right\rangle+\left\langle f, g^{\prime}\right\rangle,\langle f, g\rangle_{, 2}=\left\langle i f^{\prime}, g\right\rangle+\left\langle f, i g^{\prime}\right\rangle,\langle f g, h\rangle=\langle g, \bar{f} h\rangle .
$$

The following results were obtained in [4].

Theorem 2.1. An oriented hypersuface $M^{n}$ in $\mathbb{R}^{n+1}, n \geq 2$ is an envelope of sphere congruence, whose other envelope is contained in the hyperplane $\Pi=\left\{\left(u_{1}, u_{2}, \cdots, u_{n+1}\right) \in \mathbb{R}^{n+1}: u_{n+1}=0\right\}$ if, and only if, exist an orthogonal local parametrization of $\Pi, Y: U \subset \mathbb{R}^{n} \rightarrow \Pi$ and a differentiable function $h: U \subset \mathbb{R}^{n} \rightarrow \mathbb{R}$, such that $X: U \subset \mathbb{R}^{n} \rightarrow M^{n}$, given by

$$
X(u)=Y(u)-\frac{2 h(u)}{S}\left[\sum_{j=1}^{n} \frac{h_{, j}}{L_{j j}} Y_{, j}-e_{n+1}\right],
$$


is a parametrization of $M^{n}$, with $e_{n+1}=(0,0, \ldots, 0,1), L_{i j}=\left\langle Y_{, i}, Y_{, j}\right\rangle 1 \leq i, j \leq n$ and

$$
S=\sum_{j=1}^{n} \frac{\left(h_{, j}\right)^{2}}{L_{j j}}+1 .
$$

Moreover, the Gauss map is given by

$$
N(u)=e_{n+1}+\frac{2}{S}\left[\sum_{j=1}^{n} \frac{h_{, j}}{L_{j j}} Y_{, j}-e_{n+1}\right],
$$

and the Weingarten matrix is given by

$$
W=2 V(S I-2 h V)^{-1}
$$

where the matrix $V=\left(V_{i j}\right)$ is given by

$$
V_{i j}=\frac{1}{L_{j j}}\left(h_{, i j}-\sum_{l=1}^{n} \widetilde{\Gamma}_{i j}^{l} h_{, l}\right), 1 \leq i, j \leq n,
$$

where $\widetilde{\Gamma}_{k i}^{l}$ are the Christoffel symbols of the metric $L_{i j}$.

The regularity condition of $X$ is given by

$$
P=\operatorname{det}(S I-2 h V) \neq 0 .
$$

Corollary 2.1. With the conditions of the Theorem 2.3, we have that the first, second and third fundamental forms are given by

$$
\begin{aligned}
I & =L-\frac{2 h}{S}\left((V L)^{T}+V L\right)+\left(\frac{2 h}{S}\right)^{2} V L V^{T}, \\
I I & =-\frac{2}{S}(V L)^{T}+\frac{4 h}{S^{2}} V L V^{T}, \\
I I I & =\frac{4}{S^{2}} V L V^{T},
\end{aligned}
$$

where $L$ is the matrix of the metric $\left(L_{i j}\right)$ and $T$ denotes the transpose.

Corollary 2.2. Let $X: U \subset \mathbb{R}^{n} \rightarrow M^{n} \subset \mathbb{R}^{n+1}$ be a parametrization of the hypersurface $M^{n}$ given by (2.7). Then the following conditions are equivalent

- $X$ is parametrized by lines of curvature,

- $V_{i j}=0$ for $i \neq j$,

- $N_{, i}=-k_{i} X_{, i}$

where $k_{i}, 1 \leq i \leq n$, are the principal curvatures of $X$.

Remark 2.1. From (2.10), the principal curvatures are given by

$$
k_{i}=\frac{2 V_{i i}}{2 h V_{i i}-S}, \quad 1 \leq i \leq n .
$$

3. Surfaces with planar lines of curvature. The following result is a consequence of Theorem 2.3 , for $n=2$.

Theorem 3.1. Let $M^{2}$ be an oriented surface as in Theorem $2.3, Y(u)=(g(u), 0)$, an orthogonal local parametrization of $\Pi=\left\{\left(u_{1}, u_{2}, u_{3}\right) \in \mathbb{R}^{3}: u_{3}=0\right\}, g: U \subset \mathbb{C} \rightarrow \mathbb{C}$ is a holomorphic function with $\left|g^{\prime}\right| \neq 0$. Then there exist a differentiable function $h: U \subset \mathbb{R}^{2} \rightarrow \mathbb{R}$, such that $M^{2}$ can be locally parametrized by

$$
X(u)=(g, 0)-\frac{2 h}{S}\left(\frac{g^{\prime} \cdot \nabla h}{\left|g^{\prime}\right|^{2}},-1\right),
$$


where $u=\left(u_{1}, u_{2}\right) \in U \subset \mathbb{R}^{2}$,

$$
S=\frac{|\nabla h|^{2}}{\left|g^{\prime}\right|^{2}}+1
$$

The Gauss map is given by

$$
N(u)=\left(0,0,1-\frac{2}{S}\right)+\frac{2}{S}\left(\frac{g^{\prime} \cdot \nabla h}{\left|g^{\prime}\right|^{2}}, 0\right) .
$$

Moreover, the coefficients of the first, second and third fundamental form of $X$ are given respectively by

$$
\begin{gathered}
a_{11}=\left|g^{\prime}\right|^{2}\left(1-\frac{4 h}{S} V_{11}+\left(\frac{2 h}{S}\right)^{2}\left(V_{11}^{2}+V_{12}^{2}\right)\right) \\
a_{12}=\left|g^{\prime}\right|^{2} V_{12}\left(-\frac{4 h}{S}+\left(\frac{2 h}{S}\right)^{2}\left(V_{11}+V_{22}\right)\right), \\
a_{22}=\left|g^{\prime}\right|^{2}\left(1-\frac{4 h}{S} V_{22}+\left(\frac{2 h}{S}\right)^{2}\left(V_{12}^{2}+V_{22}^{2}\right)\right), \\
b_{11}=\left|g^{\prime}\right|^{2}\left(-\frac{2}{S} V_{11}+\frac{4 h}{S}\left(V_{11}^{2}+V_{12}^{2}\right)\right) \\
b_{12}=\left|g^{\prime}\right|^{2} V_{12}\left(-\frac{2}{S}+\frac{4 h}{S}\left(V_{11}+V_{22}\right)\right), \\
b_{22}=\left|g^{\prime}\right|^{2}\left(-\frac{2}{S} V_{22}+\frac{4 h}{S}\left(V_{12}^{2}+V_{22}^{2}\right)\right) \\
c_{11}=\frac{4}{S^{2}}\left|g^{\prime}\right|^{2}\left(V_{11}^{2}+V_{12}^{2}\right), c_{12}=\frac{4}{S^{2}}\left|g^{\prime}\right|^{2} V_{12}\left(V_{11}+V_{22}\right), c_{22}=\frac{4}{S^{2}}\left|g^{\prime}\right|^{2}\left(V_{12}^{2}+V_{22}^{2}\right),
\end{gathered}
$$

where

$$
\begin{aligned}
& V_{11}=\frac{1}{\left|g^{\prime}\right|^{2}}\left[h_{, 11}-\left\langle\frac{g^{\prime \prime}}{g^{\prime}}, \nabla h\right\rangle\right], \\
& V_{12}=\frac{1}{\left|g^{\prime}\right|^{2}}\left[h_{, 12}-\left\langle i \frac{g^{\prime \prime}}{g^{\prime}}, \nabla h\right\rangle\right], \\
& V_{22}=\frac{1}{\left|g^{\prime}\right|^{2}}\left[h_{, 22}+\left\langle\frac{g^{\prime \prime}}{g^{\prime}}, \nabla h\right\rangle\right] .
\end{aligned}
$$

Proof: For $n=2$, from Theorem 2.3, let $Y(u)=(g(u), 0)$ be a orthogonal locally parametrization of $\Pi=\left\{\left(u_{1}, u_{2}, u_{3}\right) \in \mathbb{R}^{3}: u_{3}=0\right\}$. Thus, we obtain that, $L_{11}=L_{22}=\left|g^{\prime}\right|^{2}$ and by equation (2.8), we get (3.2). Using (2.7) we obtain (3.1).

Also, from Corollary 2.4 we obtain (3.4), (3.5) and (3.6).

Finally, (3.7) it follows from (2.11) and the fact that

$$
\widetilde{\Gamma}_{i i}^{i}=\frac{\left|g^{\prime}\right|_{, i}}{\left|g^{\prime}\right|}, \quad \widetilde{\Gamma}_{i j}^{i}=\frac{\left|g^{\prime}\right|_{, j}}{\left|g^{\prime}\right|}=-\widetilde{\Gamma}_{i i}^{j}, 1 \leq i \neq j \leq 2 .
$$

The proof is complete.

The following result provides a characterization of the surfaces parametrized by planar lines of curvature.

Lemma 3.1. Let $X: U \subset \mathbb{R}^{2} \rightarrow M^{2} \subset \mathbb{R}^{3}$ be a parametrization of the surface $M^{2}$ by lines of curvature with two distinct principal curvatures. Then the lines of curvature are planar if, and only if

$$
k_{i, i} k_{i, j}\left(k_{j}-2 k_{i}\right)=k_{i, j i} k_{i}\left(k_{j}-k_{i}\right), 1 \leq i \neq j \leq 2 .
$$


Proof: By Proposition 2.2, $M^{2}$ has planar curvature lines if, and only if

$$
k_{i, i} \Gamma_{i j}^{i}+k_{i} m_{i j}=0,1 \leq i \neq j \leq 2 .
$$

Using (2.3) and (2.4) in the above expression we get that

$$
\begin{aligned}
\frac{k_{i, i} k_{i, j}}{k_{j}-k_{i}} & =k_{i}\left[\frac{k_{i, j i}\left(k_{j}-k_{i}\right)-k_{i, j}\left(k_{j, i}-k_{i, i}\right)}{\left(k_{j}-k_{i}\right)^{2}}+\frac{k_{i, j} k_{j, i}}{\left(k_{j}-k_{i}\right)^{2}}\right], \\
\frac{k_{i, i} k_{i, j}}{k_{j}-k_{i}} & =k_{i}\left[\frac{k_{i, j i}\left(k_{j}-k_{i}\right)+k_{i, j} k_{i, i}}{\left(k_{j}-k_{i}\right)^{2}}\right] .
\end{aligned}
$$

Therefore, the last equation is equivalent to (3.9). The proof is complete.

Lemma 3.2. Let $Y: U \subset \mathbb{R}^{2} \rightarrow \Pi$ be a parametrization of the plane $\Pi=\left\{\left(u_{1}, u_{2}, u_{3}\right): u_{3}=0\right\}$ given by $Y(u)=(g(u), 0), g: U \subset \mathbb{C} \rightarrow \mathbb{C}$ a holomorphic function, $u=\left(u_{1}, u_{2}\right) \in U$ and $h: U \rightarrow \mathbb{R} a$ differentiable function.

If the immersion $X: U \rightarrow \mathbb{R}^{3}$ given by (3.1) is parametrized by lines of curvature. Then for $1 \leq i \neq j \leq 2$

$$
\begin{gathered}
k_{i, i}=-\frac{2 S V_{i i, i}}{\left(2 h V_{i i}-S\right)^{2}}, \\
k_{i, j}=-\frac{2 V_{i i, j} S+4 h, j V_{i i}\left(V_{i i}-V_{j j}\right)}{\left(2 h V_{i i}-S\right)^{2}}, \\
k_{i, j i}=\frac{8 h, j V_{i i} V_{i i, i}\left(S-h V_{j j}\right)+4 S V_{i i, i}\left(2 h V_{i i, j}-h, j V_{j j}\right)+2 S V_{i i, i j}\left(S-2 h V_{i i}\right)}{\left(2 h V_{i i}-S\right)^{3}} .
\end{gathered}
$$

Proof: Since $X$ is parametrized by lines of curvature then the principal curvatures are given by (2.16) and differentiating this expression with relation to $u_{i}$ and $u_{j}$, we get respectively

$$
\begin{aligned}
k_{i, i}= & \frac{2 V_{i i, i}\left(2 h V_{i i}-S\right)-2 V_{i i}\left(2 h_{, i} V_{i i}+2 h V_{i i, i}-S_{, i}\right)}{\left(2 h V_{i i}-S\right)^{2}}, \\
k_{i, j}= & \frac{2 V_{i i, j}\left(2 h V_{i i}-S\right)-2 V_{i i}\left(2 h, j V_{i i}+2 h V_{i i, j}-S_{, j}\right)}{\left(2 h V_{i i}-S\right)^{2}} .
\end{aligned}
$$

Also, from (3.2)

$$
\begin{aligned}
S_{, i} & =\left(\frac{\left(h_{, i}\right)^{2}+\left(h_{, j}\right)^{2}}{\left|g^{\prime}\right|^{2}}+1\right)_{, i}, \\
& =\frac{\left(2 h_{, i} h_{, i i}+2 h_{, j} h_{, j i}\right)\left|g^{\prime}\right|^{2}-\left(h_{, i}^{2}+h_{, j}^{2}\right)\left|g^{\prime}\right|_{, i}^{2}}{\left|g^{\prime}\right|^{4}}
\end{aligned}
$$

where $\left|g^{\prime}\right|_{, i}^{2}$ is equal to $2\left\langle g^{\prime \prime}, g^{\prime}\right\rangle$ or $2\left\langle i g^{\prime \prime}, g^{\prime}\right\rangle$.

Thus, using (2.6)

$$
S_{, i}=\frac{1}{\left|g^{\prime}\right|^{2}}\left(2 h_{, i} h_{, i i}-2 \frac{\left\langle g^{\prime \prime}, g^{\prime}\right\rangle}{\left|g^{\prime}\right|^{2}} h_{, i}^{2}+2 h_{, j} h_{, i j}-2 \frac{\left\langle g^{\prime \prime}, g^{\prime}\right\rangle}{\left|g^{\prime}\right|^{2}} h_{, j}^{2}\right)
$$

or

$$
S_{, i}=\frac{1}{\left|g^{\prime}\right|^{2}}\left(2 h_{, i} h_{, i i}-2 \frac{\left\langle i g^{\prime \prime}, g^{\prime}\right\rangle}{\left|g^{\prime}\right|^{2}} h_{, i}^{2}+2 h_{, j} h_{, i j}-2 \frac{\left\langle i g^{\prime \prime}, g^{\prime}\right\rangle}{\left|g^{\prime}\right|^{2}} h_{, j}^{2}\right)
$$

Now, using the fact that $V_{i j}=V_{j i}=0$, we have

$$
S_{, i}=2 V_{i i} h_{, i}, i=1,2 .
$$

Substituting (3.15) in the expressions (3.13) and (3.14), we obtain (3.10) and (3.11).

Finally, differentiating (3.11) with relation to $u_{i}$ and using (3.15), we get (3.12). The proof is complete. 
The following result provides a characterization of a class of surfaces parametrized by planar lines of curvature given by (3.1).

Theorem 3.2. Let $Y: U \subset \mathbb{R}^{2} \rightarrow \Pi$ be a parametrization of the plane $\Pi=\left\{\left(u_{1}, u_{2}, u_{3}\right): u_{3}=0\right\}$ given by $Y(u)=(g(u), 0), g: U \subset \mathbb{C} \rightarrow \mathbb{C}$ a holomorphic function, $u=\left(u_{1}, u_{2}\right) \in U, h: U \rightarrow \mathbb{R} a$ differentiable function and the immersion $X: U \rightarrow \mathbb{R}^{3}$ given by (3.1) is parametrized by lines of curvature with two distinct principal curvatures.

$X$ has planar lines of curvature if, and only if,

$$
\left(2 V_{i i}-V_{j j}\right) V_{i i, i} V_{i i, j}=V_{i i}\left(V_{i i}-V_{j j}\right) V_{i i, i j}, 1 \leq i \neq j \leq 2 .
$$

Proof: Suppose that $V_{i i, j}=0$, it follows from Lemmas 3.2 and 3.3 that $X$ has planar lines of curvature if, and only if,

$$
\begin{gathered}
\frac{-2 S V_{i i, i}}{\left(2 h V_{i i}-S\right)^{2}} \frac{-4 h, j V_{i i}\left(V_{i i}-V_{j j}\right)}{\left(2 h V_{i i}-S\right)^{2}}\left(\frac{2 V_{j j}}{2 h V_{j j}-S}-\frac{4 V_{i i}}{2 h V_{i i}-S}\right)= \\
\frac{8 h, j V_{i i} V_{i i, i}\left(S-h V_{j j}\right)-4 S V_{i i, i} h_{, j} V_{j j}}{\left(2 h V_{i i}-S\right)^{3}} \frac{2 V_{i i}}{2 h V_{i i}-S}\left(\frac{2 V_{j j}}{2 h V_{j j}-S}-\frac{2 V_{i i}}{2 h V_{i i}-S}\right) .
\end{gathered}
$$

Hence,

$$
\begin{aligned}
16 S h_{, j} V_{i i} V_{i i, i}\left(V_{i i}-V_{j j}\right)\left(2 S V_{i i}-S V_{j j}-\right. & \left.2 h V_{i i} V_{j j}\right)= \\
& 16 S h_{, j} V_{i i} V_{i i, i}\left[2 V_{i i}\left(S-h V_{j j}\right)-S V_{j j}\right]\left(V_{i i}-V_{j j}\right),
\end{aligned}
$$

which is an equality.

In the case that $V_{i i, j} \neq 0$, from Lemmas 3.2 and 3.3 we get that $X$ has planar lines of curvature if, only if,

$$
\begin{aligned}
& 2 S V_{i i, i}\left(2 S V_{i i, j}+4 h_{j} V_{i i}\left(V_{i i}-V_{j j}\right)\right)\left(4 S V_{i i}-2 S V_{j j}-4 h V_{i i} V_{j j}\right)= \\
& \quad 4 S V_{i i}\left(V_{i i}-V_{j j}\right)\left(8 h, j V_{i i} V_{i i, i}\left(S-h V_{j j}\right)+4 S V_{i i, i}\left(2 h V_{i i, j}-h_{, j} V_{j j}\right)+2 S V_{i i, i j}\left(S-2 h V_{i i}\right)\right)
\end{aligned}
$$

After some calculations we get

$$
8 S^{2} V_{i i, i} V_{i i, j}\left(2 S V_{i i}-S V_{j j}-2 h V_{i i} V_{j j}\right)=8 S^{2} V_{i i}\left(V_{i i}-V_{j j}\right)\left(4 h V_{i i, i} V_{i i, j}+V_{i i, i j}\left(S-2 h V_{i i}\right)\right) .
$$

Hence,

$$
\left(S-2 h V_{i i}\right)\left(2 V_{i i}-V_{j j}\right) V_{i i, i} V_{i i, j}=\left(S-2 h V_{i i}\right) V_{i i}\left(V_{i i}-V_{j j}\right) V_{i i, i j}
$$

this equation is equivalent to (3.16). The proof is complete.

Corollary 3.1. Let $Y: U \subset \mathbb{R}^{2} \rightarrow \Pi$ be a parametrization of the plane $\Pi=\left\{\left(u_{1}, u_{2}, u_{3}\right): u_{3}=0\right\}$ given by $Y(u)=(g(u), 0), g: U \subset \mathbb{C} \rightarrow \mathbb{C}$ a holomorphic function, $u=\left(u_{1}, u_{2}\right) \in U, h: U \rightarrow \mathbb{R} a$ differentiable function and the immersion $X: U \rightarrow \mathbb{R}^{3}$ given by (3.1) is parametrized by lines of curvature with two distinct principal curvatures.

(1) If $V_{i i, j}=0,1 \leq i \neq j \leq 2$. Then $X$ has planar lines of curvature.

(2) If $V_{11}=-V_{22}$ and $V_{i i, j}=0,1 \leq i \neq j \leq 2$. Then $X$ is a Dupin surface.

Proof: (1) It follows from Theorem 3.4.

(2) Differentiating with relation a $u_{1}$ and $u_{2}$ we have

$$
V_{11,1}=-V_{22,1}=0, V_{22,2}=-V_{11,2}=0 .
$$

Using these expressions in (3.10), we get that $k_{i, i}=0$. Therefore, $X$ is a Dupin surface. The proof is complete.

Corollary 3.2. A surface $M^{2}$ as in Theorem 3.1 parametrized by lines of curvature with

$$
h(u)=\left|z_{1}\right|\left(f_{1}\left(u_{1}\right)+f_{2}\left(u_{2}\right)\right) \text { and } g(u)=z_{1} u+z_{2}, z_{1}, z_{2} \in \mathbb{C}, z_{1} \neq 0,
$$

has planar lines of curvature.

Proof: From (3.17) and (3.7) we get

$$
V_{i i}=\frac{f_{i}^{\prime \prime}}{\left|z_{1}\right|}, i=1,2 .
$$


Consequently, $V_{i i, j}=0,1 \leq i \neq j \leq 2$ and from Corollary $3.5, M^{2}$ has planar lines of curvature. The proof is complete.

Remark 3.1. From Corollary 3.6, we obtain that:

If $g(u)=u$ and $h$ is given by (3.17) with $f_{1}\left(u_{1}\right)=c_{1} u_{1}^{2}+c_{2} u_{1}+c_{3}, f_{2}\left(u_{2}\right)=d_{1} u_{2}^{2}+d_{2} u_{2}+d_{3}, c_{i}, d_{i} \in$ $\mathbb{R}$. Then the surface $M^{2}$ is a Dupin surface, see figures 4 and 5.

Example 1 Considering $g(u)=u+1, h\left(u_{1}, u_{2}\right)=\cos u_{2}$ in Corollary 3.6, we obtain a surface parametrized by lines of curvature planar (see Fig. 1).

Here

$$
V_{11}=0, V_{22}=-\cos u_{2}
$$

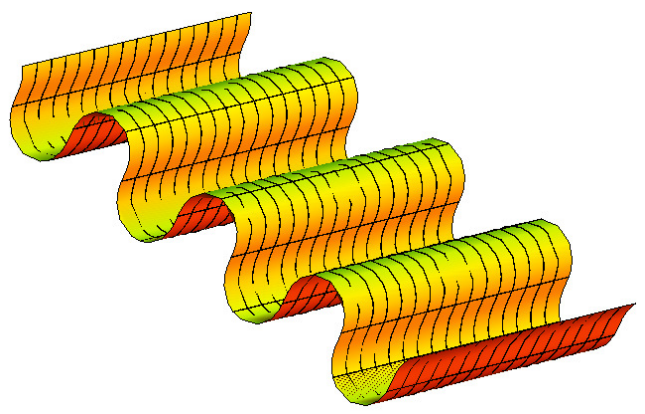

Figure 3.1: Surface with planar lines of curvature

Example 2 Considering $g(u)=(1+i) u+1+i, h\left(u_{1}, u_{2}\right)=\sqrt{2}\left(\frac{1}{100} u_{1}^{3}+\frac{1}{100} u_{1}^{2}+\cos u_{2}\right)$ in Corollary 3.6, we obtain a surface parametrized by lines of curvature planar (see Fig. 2).

Here,

$$
V_{11}=\frac{3 \sqrt{2}}{100} u_{1}+\frac{\sqrt{2}}{100}, V_{22}=-\frac{\cos u_{2}}{\sqrt{2}}
$$

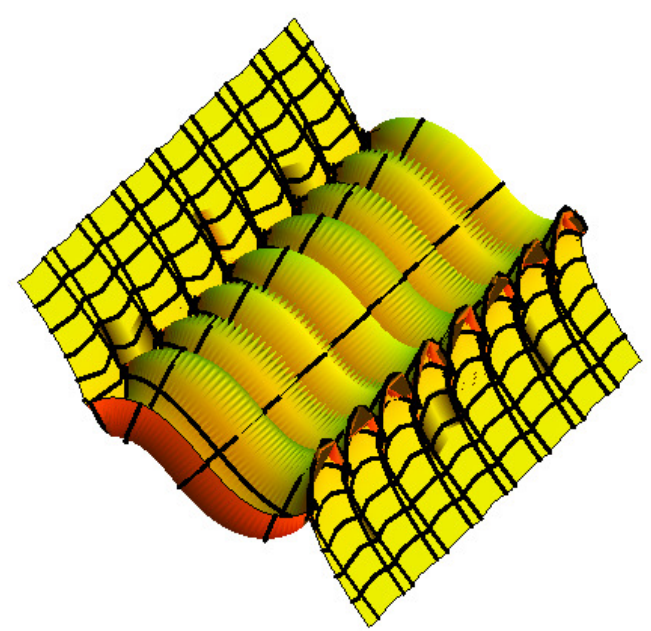

Figure 3.2: Surface with planar lines of curvature 
Example 3 Considering $g(u)=(1+i) u+1+i, h\left(u_{1}, u_{2}\right)=\sqrt{2}\left(\sin u_{1}+\cos u_{2}\right)$ in Corollary 3.6, we obtain a surface parametrized by lines of curvature planar (see Fig. 3).

Here,

$$
V_{11}=-\frac{\sin u_{1}}{\sqrt{2}}, V_{22}=-\frac{\cos u_{2}}{\sqrt{2}} .
$$

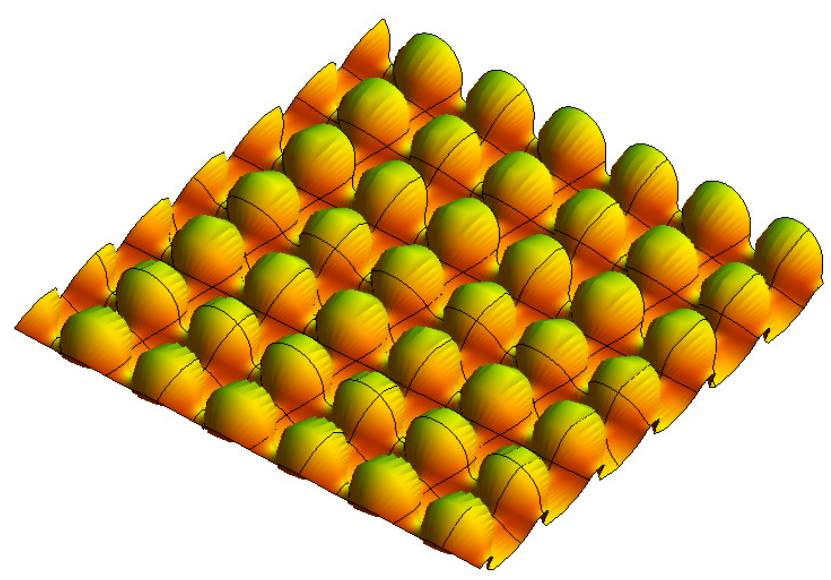

Figure 3.3: Surface with planar lines of curvature

Example 4 Considering $g(u)=u, h\left(u_{1}, u_{2}\right)=u_{2}^{2}+u_{2}+2$ in Corollary 3.6, we obtain a surface parametrized by lines of curvature planar (see Fig. 4).

Here,

$$
V_{11}=0, V_{22}=2
$$

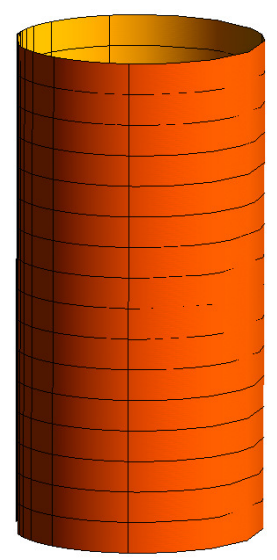

Figure 3.4: Dupin surface 
Example 5 Considering $g(u)=u, h\left(u_{1}, u_{2}\right)=-u_{1}^{2}+u_{1}+u_{2}+2$ in Corollary 3.6, we obtain a surface parametrized by lines of curvature planar (see Fig. 5).

Here,

$$
V_{11}=-2, V_{22}=0 \text {. }
$$

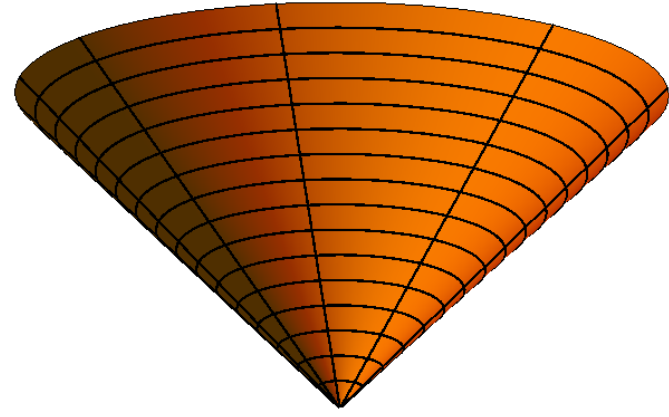

Figure 3.5: Dupin surface

Example 6 Considering $g(u)=(1+i) u, h\left(u_{1}, u_{2}\right)=\sqrt{2}\left(2 u_{1}^{2}+\cos u_{2}\right)$ in Corollary 3.6, we obtain a surface parametrized by lines of curvature planar (see Fig. 6).

$$
\text { Here, } \quad V_{11}=2 \sqrt{2}, V_{22}=-\frac{\cos u_{2}}{\sqrt{2}} \text {. }
$$

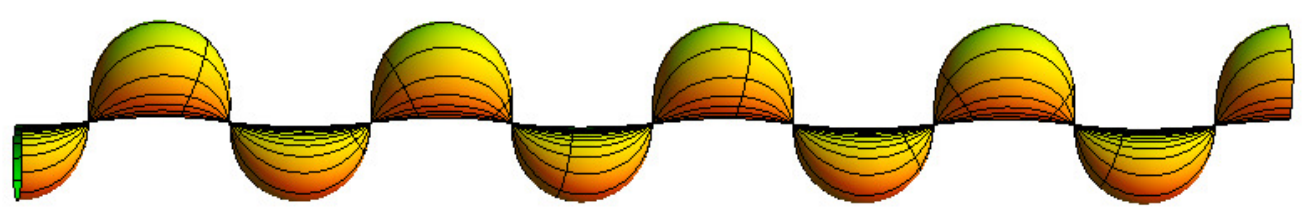

Figure 3.6: Surface with planar lines of curvature

Example 7 Considering $g(u)=(1-i) u+2-i, h\left(u_{1}, u_{2}\right)=\sqrt{2}\left(e^{u_{1}}+\sin u_{2}\right)$ in Corollary 3.6, we obtain a surface parametrized by lines of curvature planar (see Fig. 7).

Here,

$$
V_{11}=\frac{e^{u_{1}}}{\sqrt{2}}, V_{22}=-\frac{\sin u_{2}}{\sqrt{2}} .
$$

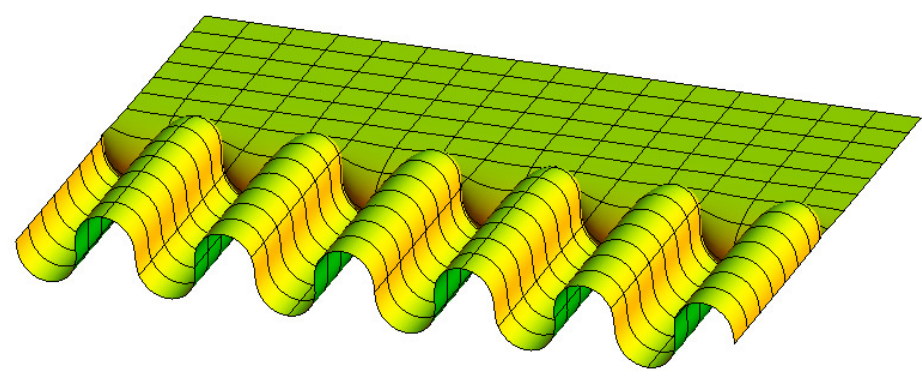

Figure 3.7: Surface with planar lines of curvature 
4. Conclusions. From the results obtained in this paper we can make the following conclusions:

We study surfaces parameterized by lines of curvature and obtain a characterization of a class of surfaces with planar lines of curvature in terms of the principal curvatures and a certain holomorphic function. Also, we present a class of surfaces with two family of planar lines of curvature, this class of surfaces includes the Dupin surfaces. Finally, the theory is illustrated with some explicit examples. It would be interesting to study surfaces parameterized by planar lines of curvature with some geometric properties such as embeddededness, completeness. In this sense, future work is being carried out.

ORCID and License

Carlos M. C. Riveros https:// orcid.org/0000-0002-1206-7072

Cid D. F. Machado https://orcid.org/0000-0003-4263-9139

This work is licensed under the Creative Commons - Attribution 4.0 International (CC BY 4.0)

\section{References}

[1] Kamran N, Tenenblat K. Laplace transformation in higher dimensions. Duke Math. Journal, 1996; 84:237-266.

[2] Kim DS, Kim YH. Surfaces with planar lines of curvature, Honam Math. 2010; 32:777-790.

[3] Leite ML. Surfaces with planar lines of curvature and orthogonal systems. J. Math. Anal. Appl. 2015; 421:1254-1273.

[4] Machado CDF, Riveros CMC. Weingarten hypersurfaces of the spherical type in Euclidean spaces. Comment. Math. Universitatis Carolinae. 2020; 61(2):213-236.

[5] Masal'tsev LA. Surfaces with planar lines of curvature in Lobachevskii space. Izv. Vyssh. Uchebn. Zaved. Math. 2001; 3:39-46.

[6] Miyaoka R. Dupin hypersurfaces and a Lie invariant. Kodai Math. J. 1989; 12:228-256.

[7] Musso E, Nicolodi L. Laguerre Geometry of Surfaces with Plane Lines of Curvature. Abh. Math. Sem. Univ. Hamburg. 1999; 69:123-138.

[8] Niebergall R. Dupin hypersurfaces in $\mathbb{R}^{5}$, Geom. Dedicata. 1991; 40:1-22, and 1992; 41:5-38.

[9] Nitsche JCC. Lectures on minimal surfaces, vol I. Cambridge University Press, 1989.

[10] Pinkall U. Dupin hypersurfaces. Math. Ann. 985; 270:427-440.

[11] Riveros CMC. Dupin hypersurfaces with four principal curvatures in $\mathbb{R}^{5}$ with principal coordinates. Rev. Mat. Complut. 2010; $23: 341-354$.

[12] Riveros CMC, Corro AMV. Invariantes de Laplace en hipersuperficies parametrizadas por líneas de curvatura. Selec. Mat. 2017; 4(1):30-37.

[13] Riveros CMC, Corro AMV. Classes of hypersurfaces with vanishing Laplace invariants. Bull. Korean Math. Soc. 2012; 49(4):685-692.

[14] Riveros CMC, Corro AMV. Hypersurfaces with planar lines of curvature in Euclidean Space. Selecciones Matemáticas. 2017; 04(02):152-161.

[15] Riveros CMC, Tenenblat K. Dupin hypersurfaces in $\mathbb{R}^{5}$. Canadian Journal of Mathematics. 2005; 57(6):1291-1313.

[16] Riveros CMC, Tenenblat K, Rodrigues LA. On Dupin hypersurfaces with constant Möbius curvature. Pacific J. Math. 2008; 236:89-103. 